\title{
EFECTO DE MUSICOTERAPIA SOBRE LA INTELIGENCIA Y LA INTERACCIÓN SOCIAL EN MUJERES PRIVADAS DE LIBERTAD CON TRASTORNOS PSIQUIÁTRICOS
}

\author{
Luis Manuel Jiménez Munguia, Luis Arturo Cruz Martínez, Lía Monserrat Vargas González, Josué \\ Edgardo Hinojosa López, Gumaro Salmerón Najera, José Ramón Silva Sánchez, J. Jesús Becerra \\ Ramírez, Obdulio Porfirio Godínez Vallejo, Yazmín Dávila Ruíz, Edith Rodríguez Meza, Jesús Eduar- \\ do Solares López, José Luis Ramos Jiménez \\ SEDESA, UNAM \\ México
}

\begin{abstract}
RESUMEN
Existe poca evidencia científica de musicoterapia en población privada de su libertad; incluso no hay datos de alguna intervención en mujeres privadas de su libertad con trastornos psiquiátricos. El objetivo del presente estudio es analizar el efecto de la musicoterapia en el factor general de inteligencia y en la interacción social en mujeres sentenciadas con trastornos psiquiátricos. Se evaluó el factor general de inteligencia de 13 participantes antes y después de una intervención de musicoterapia. Se analizó la frecuencia de conductas prosociales y agresivas emitidas durante la intervención (34 sesiones). Se encontró que las participantes tuvieron una mejoría en el factor general de inteligencia antes $(\dot{x}=18.15$, D.E. $=12.09)$ y después $(\dot{x}=21.15$, D.E. $=12.43)$ de la intervención, pero los resultados no fueron estadísticamente significativas de acuerdo con la prueba t para muestras relacionadas $(p=0.167)$. Asimismo hubo un aumento en la frecuencia de conductas prosociales y una disminución de conductas agresivas durante las sesiones. En suma, la evidencia no es contundente en la mejora del factor general de inteligencia, pero facilita la interacción social al aumentar conductas prosociales y disminuir las agresivas. Se discuten las implicaciones del estudio para futuras investigaciones.
\end{abstract}

Palabras Clave:

mujeres privadas de su libertad, musicoterapia, factor general de inteligencia, conductas prosociales, conductas agresivas.

\section{EFFECT OF MUSIC THERAPY ON INTELLIGENCE AND SOCIAL INTERACTION IN WOMEN DEPRIVED OF LIBERTY WITH PSYCHIATRIC DISORDERS}

\begin{abstract}
There is little scientific evidence of music therapy in people deprived of their liberty, there is even no evidence of any intervention with women deprived of liberty with psychiatric disorders. The objective of this study was to analyze the effect of music therapy on the general intelligence factor and on social interaction in women sentenced with psychiatric disorders. The general intelligence factor of 13 participants was evaluated before and after a music therapy intervention. The frequency of prosocial and aggressive behaviors emitted during the intervention was analyzed ( 34 sessions). It was found that the participants had an improvement in the general intelligence factor before $(\dot{x}=18.15, D E=12.09)$ and after $(\dot{x}=21.15, D E=12.43)$ of the intervention, but the results were not statistically significant according to the test $t$ for related samples $(p=.167)$. There was also an increase in the frequency of prosocial behaviors and a decrease in aggressive behaviors throughout the sessions. In sum, the evidence is not conclusive in the improvement of the general intelligence factor, but it facilitates social interaction by increasing prosocial behaviors and decreasing aggressive ones. The implications of the study for future research are discussed.
\end{abstract}

Keywords:

women deprived of their freedom, music therapy, general intelligence factor, prosocial behaviors, aggressive behaviors.

Bitácora del Artículo:

| Recibido: 1 de Febrero de 2019 | Aceptado: 1 Abril de 2019 | Publicado en línea: Enero-Junio de 2019 | 


\title{
Autoría y Derechos de Propiedad Intelectual
}

\author{
EFECTO DE MUSICOTERAPIA SOBRE LA INTELIGENCIA Y LA INTERACCIÓN SOCIAL EN \\ MUJERES PRIVADAS DE LIBERTAD CON TRASTORNOS PSIQUIÁTRICOS
}

Luis Manuel Jiménez Munguia, Luis Arturo Cruz Martínez, Lía Monserrat Vargas González, Josué Edgardo Hinojosa López, Gumaro Salmerón Najera, José Ramón Silva Sánchez, J. Jesús Becerra Ramírez, Obdulio Porfirio Godínez Vallejo, Yazmín Dávila Ruíz, Edith Rodríguez Meza, Jesús Eduardo Solares López, José Luis Ramos Jiménez SEDESA, UNAM

México

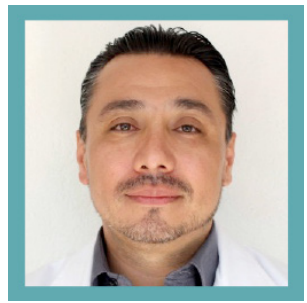

Luis Manuel Jiménez Munguia SEDESA

Correo: luismjimenezmunguia@ $\underline{\text { hotmail.com }}$

Médico cirujano en la Escuela de Medicina Tominaga Nakamoto. Maestría en Administración en la Universidad Latinoamericana. Director de Servicios Médicos Légales y en Reclusorios de la Secretaría de Salud de la Ciudad de México.

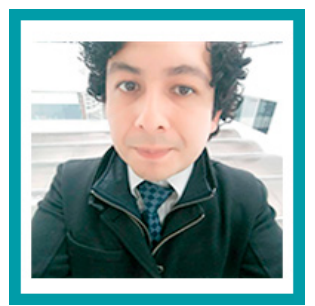

Luis Arturo Cruz Martínez SEDESA

Correo: psicomcluis23@gmail.com

Licenciado en Psicología en la Facultad de Psicología de la UNAM y psicólogo egresado del programa de Maestría en Psicología en la residencia de Medicina Conductual de la Facultad de Psicología de la UNAM.

\section{CONTRIBUCIÓN DE LOS AUTORES}

Luis Manuel Jiménez Munguia fue coordinador del proyecto | Luis Arturo Cruz Martínez hizo la elaboración del artículo, recolección de datos y análisis e interpretación de resultados | Lía Monserrat Vargas González, Josué Edgardo Hinojosa López, Gumaro Salmerón Najera y Silva Sánchez José Ramón realizaron la supervisión del trabajo de campo y recolección de datos | Jesús Becerra Ramírez, Obdulio Porfirio Godínez Vallejo, Yazmín Dávila Ruíz y Edith Rodríguez Meza fueron aplicadores de la intervención y recolección de datos | Jesús Eduardo Solares López fue el revisor del manuscrito

\section{AgRADECIMIENTOS}

Agradezco a todas aquellas personas que participaron e hicieron posible la realización de este estudio.

\section{DATOS DE FiLIACIÓN dE los Autores}

Dirección de Servicios Médico Legales y en Reclusorios, SEDESA | Facultad de Estudios Superiores Iztacala, UNAM

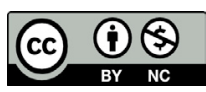

Copyright: ( 2019 Jiménez-Munguia, L.M., Cruz-Martínez, L. A., Vargas-González, L. M., Hinojosa-López, J. E., Salmerón-Najera- G., Silva-Sánchez, J. R., Becerra-Ramírez, J. J., Godínez-Vallejo, O. P., Dávila-Ruíz, Y., Rodríguez-Meza, E., Solares-López, J. E. \& Ramos-Jiménez, J. L. Este es un artículo de acceso abierto distribuido bajo los términos de la licencia Creative Commons Reconocimiento-NoComercial 4.0 Internacional, por lo que su contenido gráfico y escrito se puede compartir, copiar y redistribuir total o parcialmente sin necesidad de permiso expreso de sus autoras con la única condición de que no se puede usar con fines directamente comerciales y los términos legales de cualquier trabajo derivado deben ser los mismos que se expresan en la presente declaración. La única condición es que se cite la fuente con referencia a la Revista Digital Internacional de Psicología y Ciencia Social y a sus autoras. 


\section{TABLA DE CONTENIDO}

INTRODUCCIÓN

MÉTODO

Participantes, 24

Materiales, 25

Procedimiento, 25

Mediciones, 25

Análisis estadísticos, 26

RESULTADOS

Discusión

CONCLUSIONES

REFERENCIAS

Anexos 


\section{INTRODUCCIÓN}

\section{$\mathrm{I}$}

a musicoterapia puede ser entendida como la utilización de la música y sonidos con fines terapéuticos, que favorece el desarrollo integral de las personas, y a lo largo de su historia ha demostrado tener una importante evidencia científica en el funcionamiento cognitivo, social, emocional y físico (Love, 2017). De acuerdo con la Federación Mundial de Musicoterapia (2011), implica que su uso debe ser profesional para la intervención en ambientes médicos, educativos y cotidianos con individuos, grupos, familias o comunidades; su investigación, práctica, educación e instrucción clínica están basados en estándares profesionales según los contextos culturales, sociales y políticos.

La musicoterapia tiene una amplia gama de estudios en los que se ha encontrado que mediante estos principios las personas pueden controlar emociones (Koelsch, 2014), mejorar las habilidades de socialización (Rook, West, Wolfe, Ho, Dennis, Nakai-Hosoe y Peyton, 2014), e incluso - en niños con autismo- (Schulz, Dos Santos, Longo, Loguercio y Schüler, 2011) facilitar la creatividad (Salvador y Martínez, 2013), mejorar procesos cognitivos en población con demencia (Morrissey, Wood, Green, Pantidi y McCarthy, 2016), Alzheimer (Gómez y Gómez, 2017) o TDAH (Llamas, 2014; Salvador y Martínez, 2013), mejorar la calidad de vida en personas con trastornos mentales - como la esquizofrenia- (Mercado y Cabiya, 1991; Salvador y Martínez, 2013), e incluso hay evidencia referente a la salud mental de población penitenciaria (Fernández-Batanero y Felício, 2016). Respecto a esta población privada de su libertad, los estudios se han enfocado en objetivos como cambio en patrones de comportamiento en personalidades antisociales, disminución de conductas delictivas, disminución de abuso de sustancias, en personas con falta de red de apoyo social, aumento de conductas de autocontrol, conductas prosociales (como altruismo y cooperación), flexibilidad de la personalidad, estrategias de afrontamiento, técnicas de relajación, disminución de pensamientos y emociones negativas, manejo del enojo, aumento de empatía, manejo de impulsividad (Chen, Leith, Aarø, Manger y Gold, 2016) y manejo de la ansiedad y la depresión (Chen, Hannibal y Gold, 2016). Es necesario comentar que la evidencia en esta literatura es mucho más prevalente en población adulta que en jóvenes.
Por tanto, la musicoterapia puede ayudar a personas privadas de su libertad a regular síntomas emocionales, a socializar y a usarla como estrategia de afrontamiento; sin embargo, no hay evidencia de su efecto en funciones cognitivas que podrían verse mejoradas, sumado a estudios de musicoterapia en los que es ambigua la descripción de las mejoras psicosociales al no describirse de modo operacional a lo que se refieren dichos estudios, con conductas socialmente adecuadas o adaptativas (Fernández-Batanero y Felício, 2016; Salvador y Martínez, 2013). Por tanto, el objetivo primario del presente estudio es analizar el efecto de la musicoterapia en el factor general de inteligencia en mujeres sentenciadas con trastornos psiquiátricos, y como objetivo secundario examinar el efecto de la musicoterapia en el aumento en frecuencia de conductas prosociales (como ceder el paso, cooperar y respetar turnos) y la disminución de las conductas de agresión (por ejemplo, pegar e insultar a los demás) acorde a un registro observacional que se hizo durante las sesiones de musicoterapia.

En este estudio se espera que la musicoterapia aumente el factor general de inteligencia y la frecuencia de conductas prosociales, así como que disminuya las conductas agresivas.

\section{Método}

\section{Participantes}

Al inicio del estudio se evaluó a 39 mujeres privadas de su libertad diagnosticadas con algún trastorno psiquiátrico, de las cuales se seleccionaron 15 participantes; sólo 13 mujeres concluyeron el estudio. Los criterios de inclusión fueron que las participantes tuvieran mayoría de edad, sentencia mínima de un año respecto al inicio del estudio, que aceptaran de modo voluntario participar en el estudio, con consentimiento informado, y que obtuvieran —en el Test de Matrices Progresivas de Raven- un rango IV (inferior al término medio). Los criterios de exclusión fueron tener diagnóstico con trastorno límite de la personalidad y/o tener trastorno del comportamiento secundario al consumo de sustancias.

Al inicio del estudio las 13 participantes tuvieron una edad media de 39 años (D.E. = 9.87 años). Respecto a la escolaridad, $38.46 \%$ estudiaron el bachillerato, $15.38 \%$ concluyeron secundaria, $15.38 \%$ cursaron sólo la primaria, mientras que $30.76 \%$ no contaron con algún grado académico. Acerca de los diagnósticos de las 13 participantes, con base en la Clasificación Internacional de Enfermedades (CIE 10), 53.8\% presentaron retraso mental, $15.38 \%$ fueron diagnosticadas con algún tras- 
torno afectivo, $15.38 \%$ tuvieron trastorno mental orgánico o sintomático sin especificación, $7.69 \%$ presentaron trastorno de la personalidad y del comportamiento, y $7.69 \%$ se diagnosticaron con otros trastornos mentales consecuencia de lesión o disfunción cerebral o enfermedad somática. Es pertinente mencionar que 46.15\% de las participantes no recibieron visita, mientras que $53.85 \%$ si tuvieron visitas en el centro de reclusión por parte de sus seres queridos.

\section{Materiales}

Entrevista semiestructurada. Formatos impresos de ficha de identificación, antecedentes de salud física, mental y social, diagnóstico psiquiátrico con base en el expediente, valoración cognitiva, emocional, y conductual, valoración de consumo de sustancias y de intereses musicales (anexo 2).

Test de Matrices Progresivas de Raven. Para medir el factor general de inteligencia se utilizaron cuadernillos y formatos de respuesta (anexo 3).

Registros observacionales. Formatos impresos para medir la frecuencia de conductas prosociales y agresivas. Los registros observacionales fueron llenados por dos investigadores a quienes se les capacitó acerca de qué elementos considerar para registrar las conductas prosociales (ceder el paso, decir buenos días, organizar sillas para las dinámicas, apoyar a sus compañeras físicamente - cargando cosas, ayudando a caminar o ayudando a mover a una participante que se trasladaba en silla de ruedas - y reforzadores sociales — "tú puedes", "lo hiciste muy bien" - etcétera) y para registrar las conductas agresivas (insultar a alguien, pegar a algo o alguien, interrumpir la participación de alguien) (anexo 4).

Materiales para la fase de tratamiento. Se utilizaron dos guitarras, dos bongos, tarjetas de trabajo con diversas imágenes y frases propias de las necesidades de cada sesión.

\section{Procedimiento}

Para el desarrollo del presente estudio se obtuvo la autorización del Comité de Ética de la dirección de enseñanza de la Secretaría de Salud. Posterior al consentimiento, se desarrolló el estudio, que constó de tres fases: 1) evaluación pretratamiento; 2) tratamiento, y 3) evaluación postratamiento.

Se inició con la fase de evaluación pretratamiento que consistió en evaluar - a través de evaluaciones del Test de matrices Progresivas de Raven y entrevistas semiestructuradas individuales-y seleccionar a 15 de 39 mujeres con trastorno psiquiátrico privadas de su libertad.

El tratamiento se dividió en seis bloques, cada uno de los cuales se enfocó en la ejecución de diferentes actividades con objetivos específicos. Cabe mencionar que cada bloque estuvo conformado por cinco o seis sesiones, y cada una de ellas tuvo una duración de una hora y media. En el primer bloque (cinco sesiones) se contemplaron habilidades sociales de integración y cohesión grupal, en un entorno de confianza, juego y seguridad entre las participantes. En el segundo bloque (seis sesiones) se requirió de sincronía y precisión para generar melodías, lo que fomentó la atención y el aprendizaje dada la secuenciación. En el tercer bloque (seis sesiones) se fomentó la creatividad, que implicó un nivel elevado de atención. En el cuarto bloque (cinco sesiones) se reforzó la concentración, el aprendizaje y la secuenciación por medio de dinámicas diferentes a las del segundo bloque. El quinto bloque (seis sesiones) tuvo como objetivo condicionar a las participantes a no efectuar conductas que dañen la interacción social y facilitar bajos niveles de hostilidad y altos niveles de atención a conductas prosociales. El sexto bloque (seis sesiones) se enfocó en el desarrollo de conductas prosociales; en él se trabajaron también el aprendizaje y la atención.

La fase de evaluación postratamiento se brindó tres meses después de la última sesión de tratamiento. A las participantes se les evaluó de nuevo con el Test de Matrices Progresivas de Raven, para verificar la eficacia de tratamiento, comparando el antes y el después de la intervención, como sucede en los diseños pre-post tratamiento.

\section{Mediciones}

Por medio de una entrevista semiestructurada se recolectaron datos sociodemográficos (como edad, fecha de nacimiento, estado civil, tiempo de estancia en el centro de reclusión, tiempo restante de sentencia, ocupación en el centro, información referente al interés por el tratamiento de musicoterapia y diagnóstico psiquiátrico corroborado por expediente clínico de psiquiatría).

Se utilizó el Test de Matrices Progresivas de Raven para medir el factor general de inteligencia según la teoría de Charles Spearman. Se trata de una prueba de elección múltiple, no cultural, ya que no intervienen los Conocimientos adquiridos; por ello, el grado académico no es determinante para su aplicación; es no verbal; puede ser aplicado a cualquier persona sin importar idioma, educación o capacidad verbal; incluso, se puede aplicar a personas con analfabetismo y a personas sordomudas. Consiste en la presentación de 60 matrices acomodadas en orden de dificultad creciente. Se pretende que el participante utilice sus habilidades de percepción, de observación y razonamiento para deducir la pieza faltante en la matriz. La puntuación obtenida por el sujeto se relaciona con un grupo de 100 sujetos de su misma 
edad para determinar el percentil, y así poder asignarlo a un rango (95 o más: superioridad intelectual; 90 y 75 : superior al término medio; 50: término medio; 25 y 10: inferior al término medio, y 5: deficiencia intelectual). La prueba tiene una confiabilidad de 0.87 a 0.81 y su validez, según el criterio de Terman Merril, alcanza un índice de 0.86 .

Mediante registros observacionales, dos investigadores contabilizaron la frecuencia de conductas prosociales (ceder el paso, cooperar, respetar indicaciones y turnos) y la frecuencia de conductas agresivas (pegar, insultar o gritar a las demás participantes o musicoterapeutas, no respetar turnos o indicaciones) de las participantes durante cada sesión.

\section{Análisis estadísticos}

Para el presente estudio se utilizaron diferentes análisis. Uno de ellos fue el uso de la Prueba T para muestras relacionadas, ya que es el análisis estadístico fue sugerido para comparar las medias del pre y post tratamiento en un solo grupo de participantes (Pagano, 1999), que fue el caso del presente estudio (las medias referentes al factor general de inteligencia del grupo de participantes).

Mediante los registros observacionales se midieron las frecuencias por sesión de las conductas prosociales y de agresión que las participantes manifestaron durante cada una de las sesiones. El análisis de estos datos se hizo por medio del cambio clínico objetivo de Cardiel, que mide la magnitud del cambio del tratamiento en función de la condición inicial y la condición final (Cormier, Nurius y Osborn, 2016). Sólo cambios superiores a 0.20 (una quinta parte de cambio del nivel inicial) son clínicamente significativos. La fórmula es CCO = Condición final - Condición inicial / Condición inicial. Concerniente al presente estudio, la fórmula se despejará considerando la media de frecuencia de conductas prosociales del sexto bloque (condición final) menos la media de frecuencias de dichas conductas del primer bloque (condición inicial) entre la media de frecuencias del primer bloque (condición inicial), mientras que para las conductas agresivas se despejaría al revés, porque se busca que el índice de frecuencias, es decir, considerando al primer bloque como la condición final y al sexto bloque como la condición inicial.

\section{Resultados}

De 15 participantes, 13 concluyeron el estudio porque una participante decidió de manera voluntaria retirarse en la sesión 4 del tratamiento y otra tuvo siete faltas.
De 34 sesiones de intervención con musicoterapia, las participantes asistieron a un promedio de 29.5 sesiones $(86.7 \%)$.

En la tabla 1 se muestra el análisis de las medias y prueba t con base en los índices obtenidos por las 13 participantes del presente estudio en el Test de Matrices Progresivas de Raven, antes $(\dot{x}=18.15$, D.E. $=12.09)$ y después $(\dot{x}=21.15$, D.E. $=12.43$ ) de la intervención. Se observa un aumento en el porcentaje obtenido en el test por parte de las participantes $(6.28 \%)$, pero no son estadísticamente significativas $(p=0.167)$ con base en el análisis de la prueba t.

Tabla 1. Puntuaciones en Test de Matrices Progresivas de Raven antes y después de la intervención.

\begin{tabular}{lll} 
& Pre-test & Post-test \\
\hline $\mathrm{N}=13$ & 18.15 & 21.92 \\
$\begin{array}{l}\text { Media de puntajes } \\
\text { obtenidos }\end{array}$ & 12.43 \\
$\begin{array}{l}\mathrm{D} \text { e s v i a c i ó } \mathrm{n} \\
\text { estándar }\end{array}$ & 12.09 & \\
$\begin{array}{l}\text { Puntos obtenidos } \\
\text { (\%) }\end{array}$ & 30.26 & \\
$\begin{array}{l}\text { Grados de libertad } \\
\text { (gl) }\end{array}$ & 12.00 & \\
Valor crítico de $t$ & 1.782 & \\
$\mathrm{P}=0.167$ & & \\
\hline
\end{tabular}

En la figura 1 se muestran, en el eje de las ordenadas, las frecuencias de las conductas prosociales (como ceder el paso, cooperar, respetar turnos) y las conductas agresivas (no respetar turnos, gritar, pegar, insultar o burlarse de los demás), emitidas por las 13 participantes durante las 34 sesiones (eje de las abscisas) de la intervención. Las líneas verticales dividen los bloques con que se trabajó (bloque 1, de habilidades sociales y cohesión grupal; bloques 2 y 4 , de atención y aprendizaje mediante sincronía y precisión; bloque 3, de creatividad; bloques 5 y 6 , de reforzamiento de conductas prosociales y eliminación de conductas agresivas). Se observa que en el primer bloque la frecuencia de las conductas prosociales fue menor que las agresivas; sin embargo, a partir del segundo bloque se percibe un aumento en la frecuencia de conductas prosociales y una disminución en las conductas agresivas, manteniéndose estable la tendencia de las frecuencias en el quinto y el sexto bloques. 


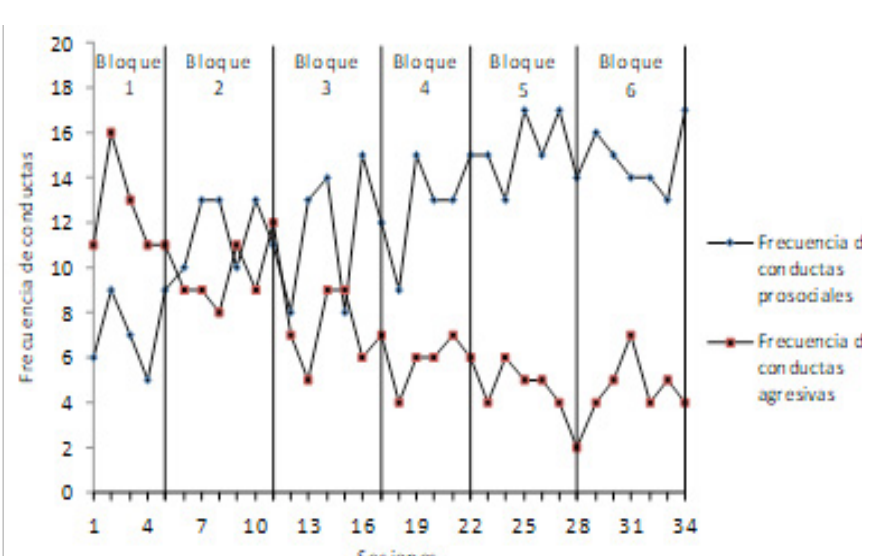

Figura 1. Resultados de frecuencias de conductas prosociales y agresivas.

En la figura 2 se muestra el análisis del cambio clínico objetivo. De acuerdo con la fórmula $\mathrm{CCO}=$ Condición final - Condición inicial / Condición inicial, se encontró que para el cambio clínico objetivo en las conductas prosociales (barra azul) fue de 1.06, mientras que el cambio clínico objetivo para las conductas agresivas fue de 0.61 (barra roja). Ambos cambios fueron clínicamente objetivos al ser superiores al punto de corte, que es de 0.20 (línea azul).

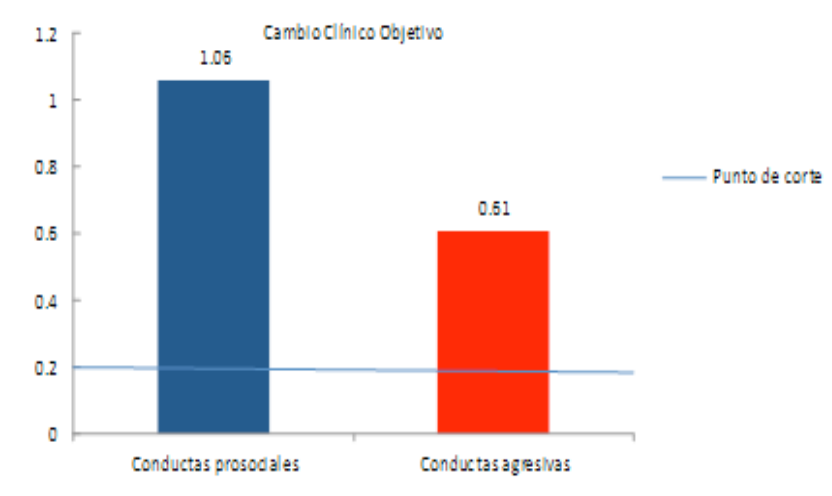

Figura 2. Puntajes de cambio clínico objetivo.

\section{Discusión}

El objetivo del presente estudio fue analizar el efecto de la musicoterapia en el factor general de inteligencia en mujeres con trastornos psiquiátricos sentenciadas, y también se analizó el efecto de la musicoterapia en el aumento en frecuencia de conductas prosociales y la disminución de las conductas de agresión.

Respecto a los índices obtenidos para medir el factor general de inteligencia, se muestra un aumento de $6.28 \%$ al comparar el antes y el después de la intervención, pero no se obtuvieron datos estadísticamente significativos. Los datos no se pueden comparar con los hallazgos obtenidos en estudios previos en los que se usó musicoterapia en población privada de su libertad, ya que éstos se enfocan en identificar su efecto en variables como manejo de emociones (Pozo, 2017; Ueda, Suzukamo, Sato y Izumi, 2013), habilidades sociales (Fernández-Batanero y Felício, 2016), además de que en estudios en los que se pretende obtener una mejora en procesos cognitivos estrechamente relacionados con el factor general de inteligencia se trabaja con personas que presentan demencia (Morrissey et al., 2016), Alzheimer (Gallego y García, 2017), dislexia o TDAH (Llamas, 2014; Salvador y Martínez, 2013), y ninguna de las participantes cumple con dichos criterios diagnósticos. Sólo en un estudio de Alzheimer (Satoh, Yuba, Tabei, Okubo, Kida, Sakuma y Tomimoto, 2015) y otro de dislexia (Forgeard, Schlaug, Norton, Rosam, lyenga y Winner, 2008) se valoró el factor general de inteligencia; la valoración utilizada en ambos estudios se efectuó con el Test de Matrices Progresivas de Raven, mientras que en el resto de los estudios sólo se consideraron procesos cognitivos particulares (como atención, lenguaje o aprendizaje), algunos de ellos son revisiones sistemáticas, pero lo hacen en personas con demencia y en combinación con otras variables (como manejo de emociones) (McDermott, Crellin, Ridder y Orrell, 2013; Ueda et al., 2013).

Respecto al análisis de frecuencias de conductas, la intervención en musicoterapia aumentó las conductas prosociales y disminuyó las agresivas; esto sugiere que la musicoterapia puede ayudar a las personas con algún diagnóstico psiquiátrico, incluyendo las que presentan enfermedades mentales graves y bajo control de impulsos. Esta evidencia es congruente con los hallazgos reportados por diferentes investigaciones, porque en un estudio en el que se intervino con musicoterapia con un grupo de cinco personas con esquizofrenia, en el que los objetivos fueron fomentar la integración del grupo y disminuir sintomatología propia de la esquizofrenia, los investigadores encontraron cambios en distintas áreas, como la sensorio-motora (mayor movilidad y coordinación corporal), la emocional (mayor expresión individual y grupal) y la social (incremento de la cohesión grupal y del sentido de la pertenencia) (Salvador y Martínez, 2013), pero esta evidencia no se sustenta con datos cuantitativos al brindar sólo un informe de satisfacción de los participantes en el estudio y un análisis narrativo de los cambios observados. El artículo que muestra mayor evidencia es el efectuado por Yang, Li, Weng y Zhang (1998) al cumplir los criterios de ensayo controlado aleatorizado acorde a dos revisiones sistemáticas (Gold, Solli, Krüger y Lie, 2009; Mössler, Chen, Heldal y Gold, 2011). Yang y colaboradores encontraron que un

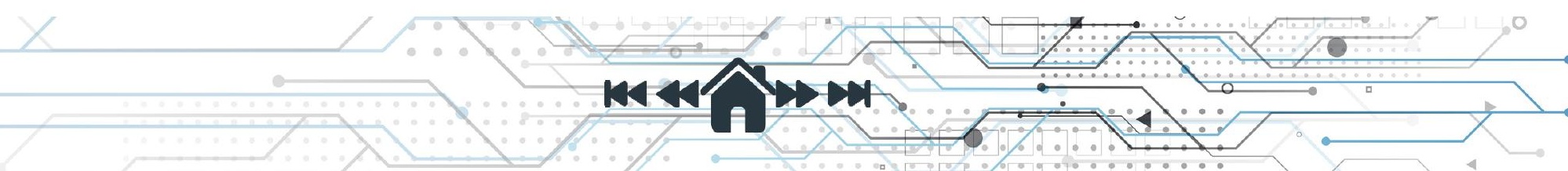


grupo de participantes con esquizofrenia con medicación psiquiátrica y musicoterapia tuvieron menor fatiga, menor embotamiento afectivo y menos pensamientos disfuncionales en comparación con un grupo de participantes con el mismo trastorno, pero sólo medicados; sin embargo, no discuten sus implicaciones psicosociales, como se hace en el presente artículo.

Lo encontrado en este estudio se añade a otros en los que se ha intervenido con musicoterapia a personas privadas de su libertad. Dichos estudios han sido analizados en una revisión sistemática que identificó que se ha estudiado el efecto de la musicoterapia en discapacidad psicosocial, manejo de emociones, conductas en personalidades antisociales disminución en frecuencia de conductas delictivas y abuso de sustancias (Chen et al., 2016), sin dejar de lado evidencia que se publicó, y que es un ensayo controlado aleatorizado en personas privadas de su libertad en China, el cual evaluó el efecto de la musicoterapia en la depresión, la ansiedad y la autoestima con resultados confiables de mejoras en estas variables de estudio, sobre todo en población joven y de bajo nivel académico (Chen et al., 2016).

Se optó por un estudio con diseño pre y post y no uno de medidas repetidas o uno de línea base múltiple que pueo ser recomendable acorde al número de participantes (Kazdin, 2011), o un ensayo controlado aleatorizado que representa un gran nivel de evidencia como el efectuado por Chen et al. (2016), por las siguientes dos razones: 1) la muestra fue pequeña porque la población también lo es, y sólo fue posible cubrir los criterios de inclusión en 15 de 34 mujeres privadas de su libertad en esta área, y 2) porque si se optaba por evaluar de manera repetida la eficiencia y eficacia de su ejecución con el Test de Matrices Progresivas de Raven se hubiese tenido un sesgo en el que las participantes hubiesen mejorado sus competencias por exponerse de modo constante a dicha prueba.

\section{Conclusiones}

Los resultados del presente estudio muestran beneficios en el aumento de conductas prosociales, disminución de las agresivas y mejoras en las competencias generales de inteligencia sin que este último resultado sea comprobable de manera científica al no encontrar diferencias significativas a nivel estadístico.

El que no haya un cambio significativo en el factor general de inteligencia tal vez se deba a que se utilizó un tipo de aprendizaje activo caracterizado por exponer a las personas a actividades que requieren socialización y extroversión en vez del tipo de aprendizaje que más usan las personas privadas de la libertad que es el teórico, porque en una investigación se mostró que de 1,150 participantes en actividades escolares en nueve centros penitenciarios de la Ciudad de México, $47 \%$ resultó preferir y aprender más fácil con un modelo teórico, 28\% con un modelo reflexivo, $16 \%$ con un modelo pragmático y $9 \%$ con un modelo activo (Castellanos, Rosas, Mora y Ruiz, 2015). Por tanto, un alcance para otras investigaciones en centros de reclusión podría enfocarse en agregar una fase teórica de la importancia de practicar musicoterapia en las habilidades cognitivas de las o los participantes para incrementar el compromiso y el apego a la intervención.

Futuros estudios en musicoterapia podrían contemplar la viabilidad de una metodología más precisa a las del presente estudio, como efectuar ensayos aleatorios controlados o si se trata de un estudio de casos únicos mediante un diseño de línea base múltiple que se ha comprobado tiene un nivel de evidencia adecuado (Vohra, Shamseer, Sampson, Bukutu, Schmid, Tate y Altman, 2015).

En México no hay evidencia para probar los efectos de una intervención en alguna variable en población privada de su libertad e incluso internacionalmente no hay algún estudio para intervenir en una población similar a las del presente estudio que presenta tres categorías diferentes de vulnerabilidad social: 1$)$ mujeres; 2) privadas de su libertad, y 3) con trastorno psiquiátrico. Lo anterior evidencia la necesidad de seguir la investigación en personas privadas en centros de reclusión y aún más en grupos vulnerables, como lo representan personas de otro país, pueblos y comunidades indígenas, población LGBTTI, niños que viven con sus madres en centros de reclusión femeniles y en comunidades de adolescentes.

\section{Referencias}

Castellanos, L. G. E., Rosas, M. M. D. E., Mora, E. V. M., \& Ruiz, O. A. H. (2015). Estilos de aprendizaje en población penitenciaria de la Ciudad de México. Journal of Learning Styles, 8(16).

Cormier, S., Nurius, P. S., \& Osborn, C. J. (2016). Interviewing and Change Strategies for Helpers. Nelson Education.

Chen, X. J., Hannibal, N., \& Gold, C. (2016). Randomized Trial of Group Music Therapy with Chinese Prisoners. Impact on Anxiety, Depression, and Self-Esteem. International Journal of Offender Therapy and Comparative Criminology, 60(9), 1064-1081. doi: 10.1177/0306624X15572795

Chen, X. J., Leith, H., Aarø, L. E., Manger, T., \& Gold, C. (2016). Music Therapy for Improving Mental Health Problems of Offenders in Correctional Settings. Systematic Review and Meta-Analysis. Journal of Experimental Criminology, 12(2), 209-228. doi 10.1007/s11292-015-9250-y 
Federación Mundial de Musicoterapia (2011), Sobre musicoterapia. Definiendo musicoterapia. Recuperado el 12 de septiembre de 2018 de https://sobre musicoterapia.wordpress.com/tag/federacion-mundialde-musicoterapia/

Fernández-Batanero, J. M., \& Felício, C.J. M. (2016). Musicoterapia e integración social en menores infractores. Un estudio de casos. Perfiles educativos, 38(152), 163-180.

Forgeard, M., Schlaug, G., Norton, A., Rosam, C., Iyengar, U., \& Winner, E. (2008). The Relation Between Music and Phonological Processing in Normal-Reading Children and Children with Dyslexia. Music Perception. An Interdisciplinary Journal, 25(4), 383-390. doi: 10.1525/ mp.2008.25.4.383

Gold, C., Solli, H. P., Krüger, V., \& Lie, S. A. (2009). Dose-Response Relationship in Music Therapy for People with Serious Mental Disorders. Systematic Review and Meta-Analysis. Clinical Psychology Review, 29(3), 193-207. doi: 10.1016/j. cpr.2009.01.001

Gómez, G. M., \& Gómez, G. J. (2017). Musicoterapia en la enfermedad de Alzheimer. Efectos cognitivos, psicológicos y conductuales. Neurología, 32(5): 300-308 doi: 10.1016/j. nrl.2015.12.003

Kazdin, A. E. (2011). Single-Case Research Designs: Methods for Clinical and Applied Settings. Oxford University Press.

Koelsch, S. (2014). Brain Correlates of Music-Evoked Emotions. Nature Reviews Neuroscience, 15(3), 170. doi: 10.1038/ $\underline{\mathrm{nrn3666}}$

Llamas, R. J. C. (2014). Alumnos con TDAH y musicoterapia. Cómo trabajar en Primaria para mejorar el desarrollo personal y escolar en estos niños. Artseduca (8), 136-157.

Love, L. W. (2017). Music Therapy. (Citado el 12 de septiembre de 2017.) http://lovewholefoods.com/ns/DisplayMonograph. DB50F17237D8\&DocID=musictherapy

McDermott, O., Crellin, N., Ridder, H. M., \& Orrell, M. (2013). Music Therapy in Dementia. A Narrative Synthesis Systematic Review. International Journal of Geriatric Psychiatry, 28(8), 781-794. doi: $10.1002 / g p s .3895$

Mercado, C. J., \& Cabiya, M. J. J. (1991). Expresión de emociones y nivel de interacción en familias de pacientes con diagnóstico de esquizofrenia. Revista Puertorriqueña de Psicología, 7(1), 6.

Morrissey, K., Wood, G., Green, D., Pantidi, N., \& McCarthy, J. (2016). I'm a Rambler, I'm a Gambler, I'm a Long Way from Home'. The Place of Props, Music, and Design in Dementia Care. Proceedings of the 2016 ACM Conference on Designing
Interactive Systems, pp. 1008-1020. ACM.

Mössler, K., Chen, X., Heldal, T. O., \& Gold, C. (2011). Music Therapy for People with Schizophrenia and SchizophreniaDlike Disorders. The Cochrane Library.

Pagano, R. R. (1999). Estadística para las ciencias del comportamiento, núm. 310 P3Y 1998.

Pozo, J. I. (2017). Learning beyond the Body. From Embodied Representations to Explicitation Mediated by External Representations/Aprender más allá del cuerpo: de las representaciones encarnadas a la explicitación mediada por representaciones externas. Infancia y Aprendizaje, 40(2), 219-276. doi: 10.1080/02103702.2017.1306942

Rook, J., West, R., Wolfe, J., Ho, P., Dennis, A., Nakai-Hosoe, Y., \& Peyton. K. (2014). Music Therapy Social Skills Assessment and Documentation Manual (MTSSA): Clinical Guidelines for Group Work with Children and Adolescents. Jessica Kingley Publishers.

Salvador, M., \& Martínez, D. (2013). Grupo de musicoterapia en un Centro de Día para personas con trastorno mental grave. Rehabilitación Psicosocial, 10(1), 30-34.

Satoh, M., Yuba, T., Tabei, K. I., Okubo, Y., Kida, H., Sakuma, H., \&Tomimoto, H. (2015). Music Therapy Using Singing Training Improves Psychomotor Speed in Patients with Alzheimer's Disease. A Neuropsychological and fMRI Study. Dementia and Geriatric Cognitive Disorders, 5(3), 296-308. doi: 10.1159/000436960

Schulz, G. G., Dos Santos, R. R.., Longo, D., Loguercio, L. J. C., \& Schüler, F. L. (2011). Effects of Relational Music Therapy on Communication of Children with Autism. A Randomized Controlled Study. Nordic Journal of Music Therapy, 20(2), 142-154. doi: $10.1080 / 08098131.2011 .566933$

Ueda, T., Suzukamo, Y., Sato, M., \& Izumi, S. I. (2013). Effects of Music Therapy on Behavioral and Psychological Symptoms of Dementia. A Systematic Review and MetaAnalysis. Ageing Research Reviews, 12(2), 628-641. doi: 10.1016/j.arr.2013.02.003

Vohra, S., Shamseer, L., Sampson, M., Bukutu, C., Schmid, C. H., Tate, R., \& Altman, D. G. (2015). CONSORT Extension for Reporting N-of-1 trials (CENT) 2015 Statement. Journal of Clinical Epidemiology. doi: https://doi.org/10.1136/bmj. $\underline{\mathrm{h} 1738}$

Yang, W. Y., Li, Z., Weng, Y. Z., Zhang, H. Y., \& Ma, B. (1998). Psychosocial Rehabilitation Effects of Music Therapy in Chronic Schizophrenia. Hong Kong Journal of Psychiatry, 8(1), 38. 
Artículo Empírico | Efecto de musicoterapia sobre la inteligencia y la interacción... | Jiménez-Munguia, et al

\section{Anexos}

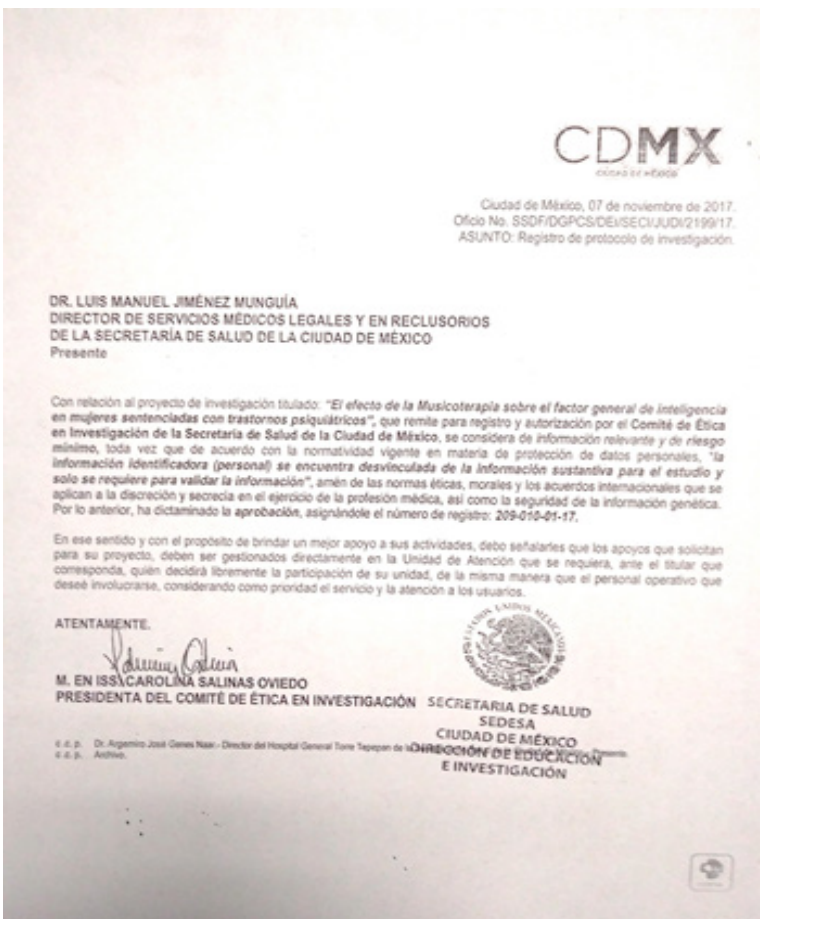

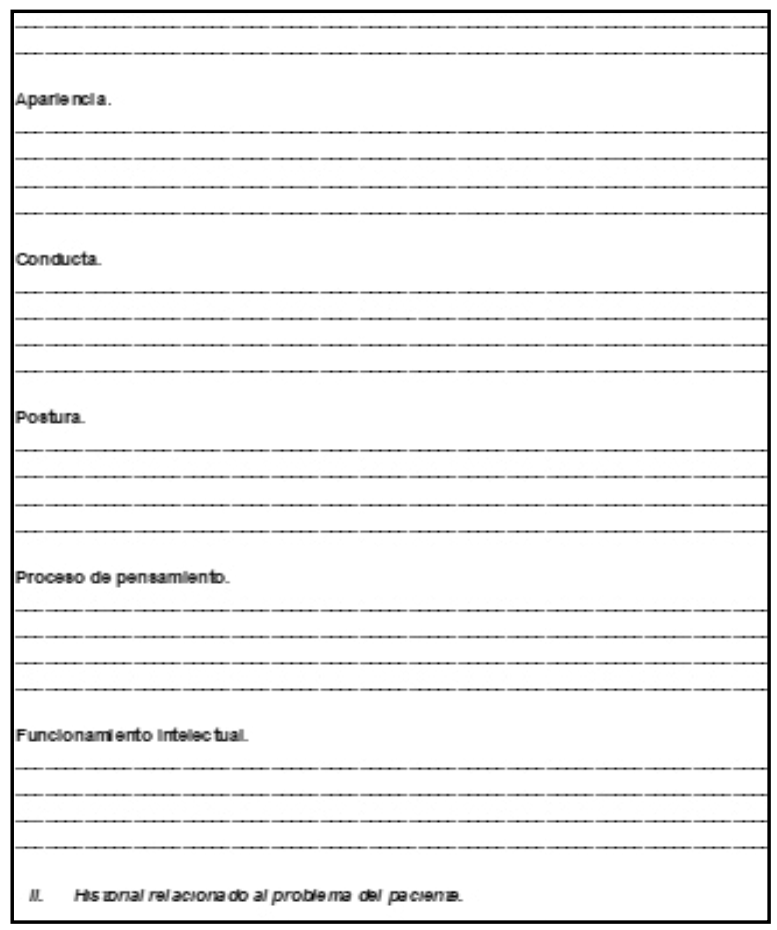

Anexo 1. Carta de aprobación de investigación elaborada por el Comité de Ética en Investigación de la Secretaría de Salud de la Ciudad de México.

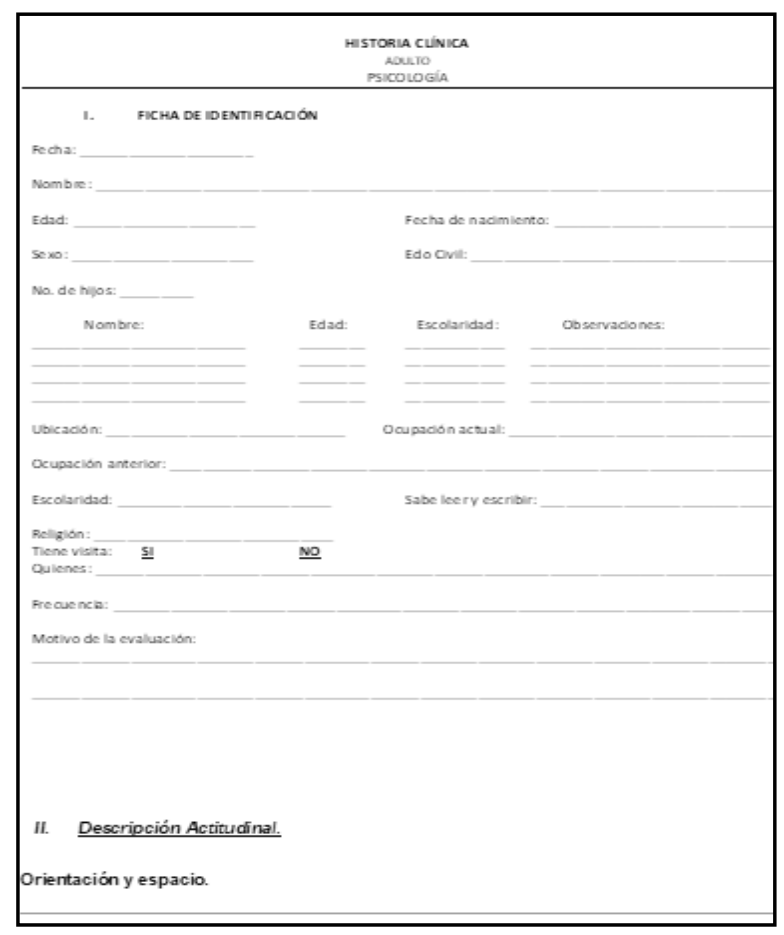




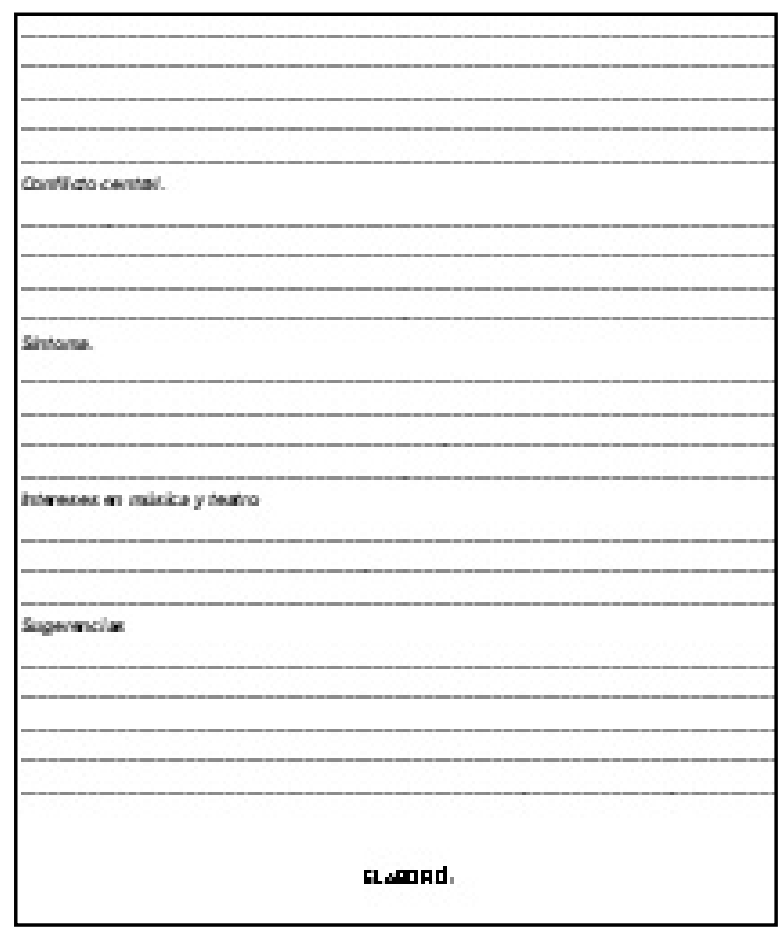

\section{Anexo 2. Historia clínica}

Instrucciones: Se te mostrarán distintas placas; tendrás que decidir cuál es la figura que completa a cada una. El test consta de 60 placas; te recomendamos que lo hagas con tiempo.

Para completar el test tienes 45 minutos; son suficientes, así que no te apures. Al finalizar obtendrás tu coeficiente intelectual con base en los valores estadísticos para tu edad.

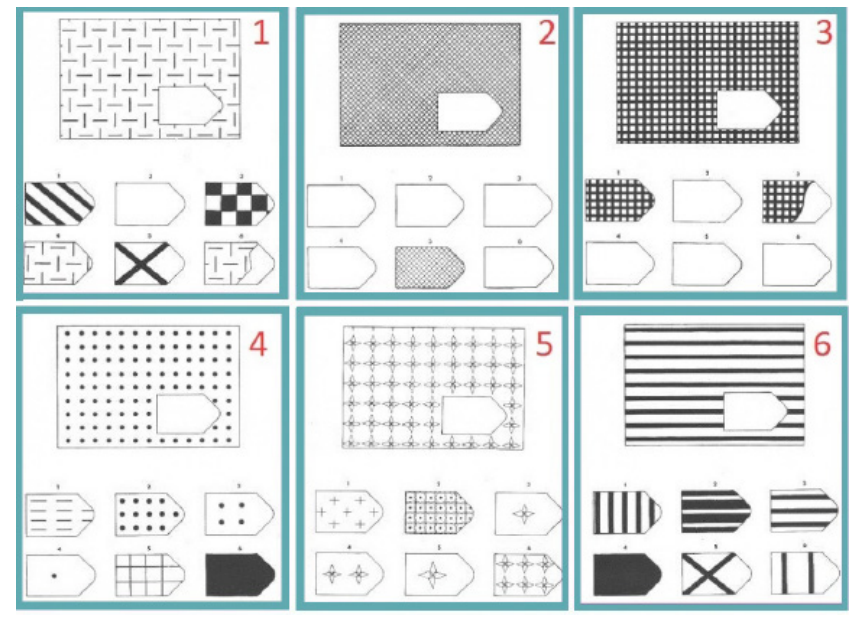

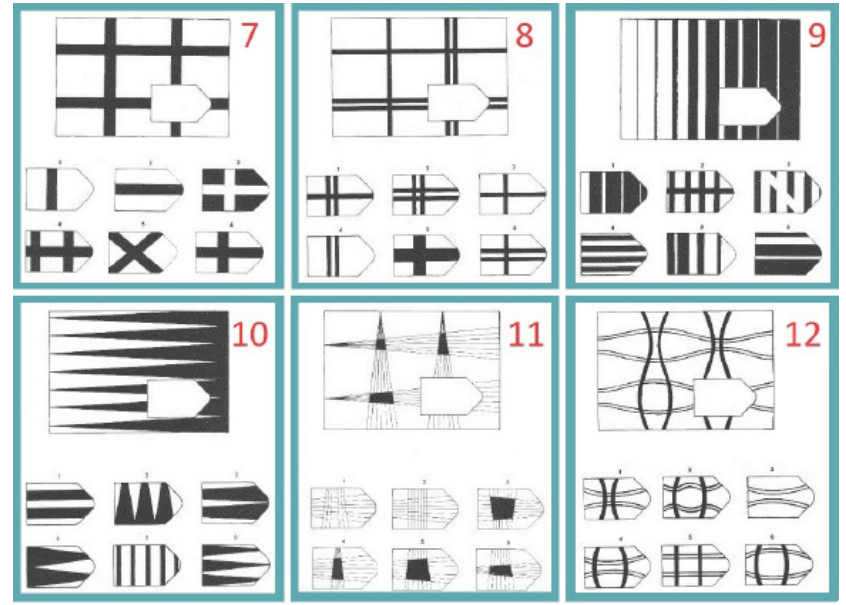

Anexo 3. Test de Matrices Progresivas RAVEN 


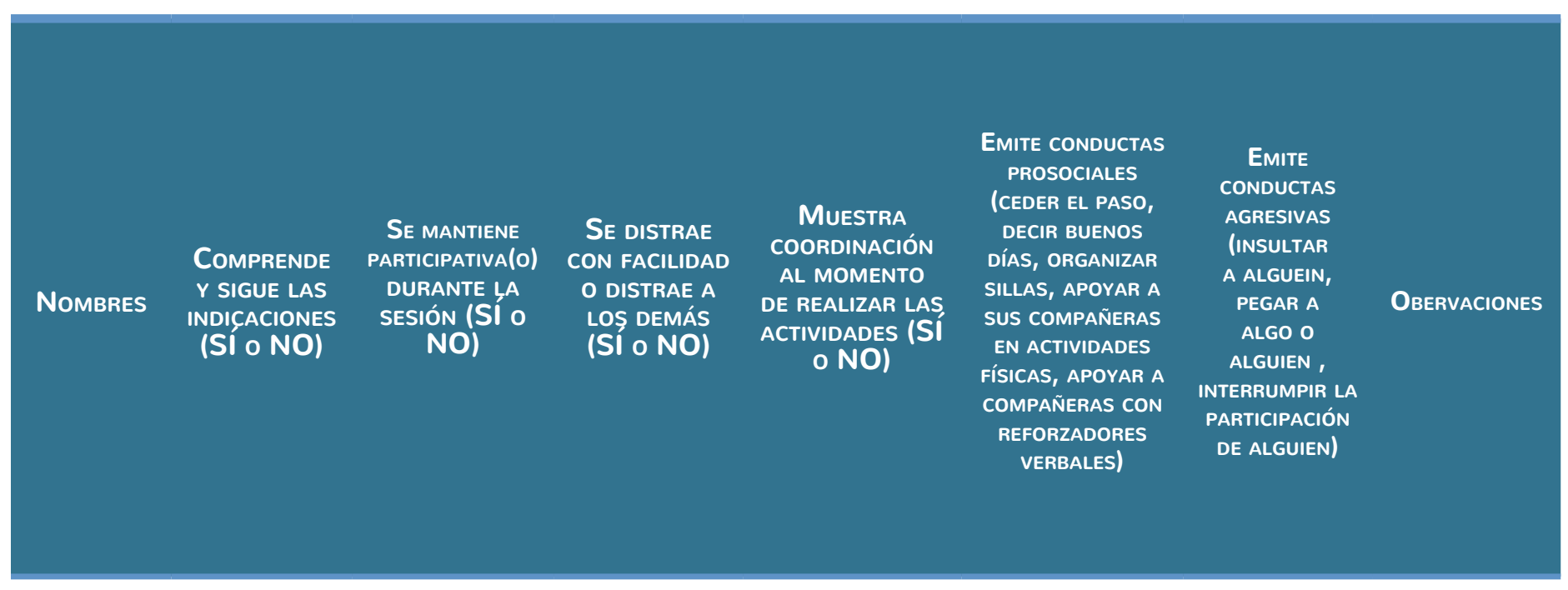

Anexo 4. Registro observacional de conductas prosociales y agresivas. 


\section{Meta-Análisis del Artículo}




\section{Dimensión Cuantitativa}

\section{Perfil de Evaluación entre pares}
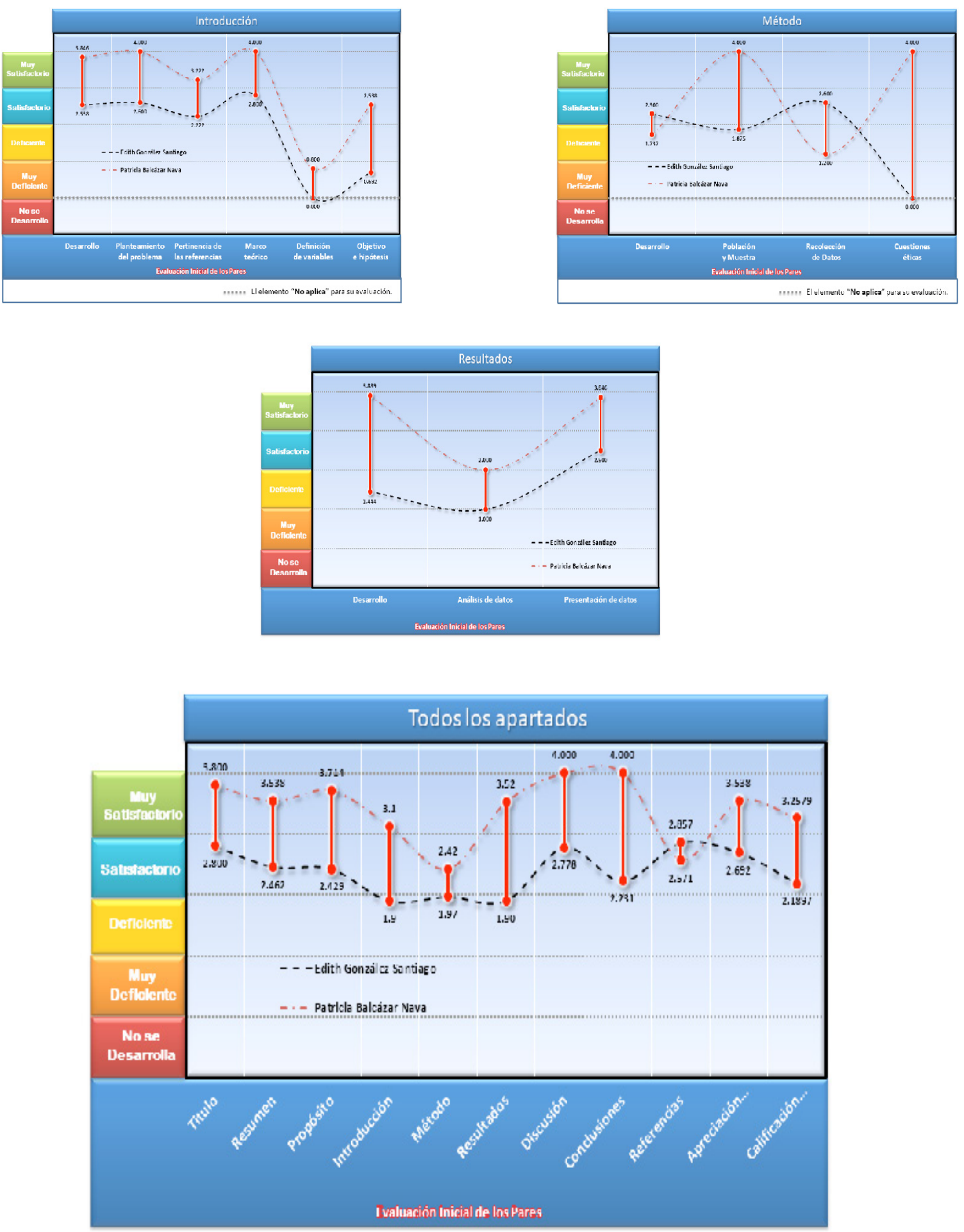


\section{Índice de Concordancia}

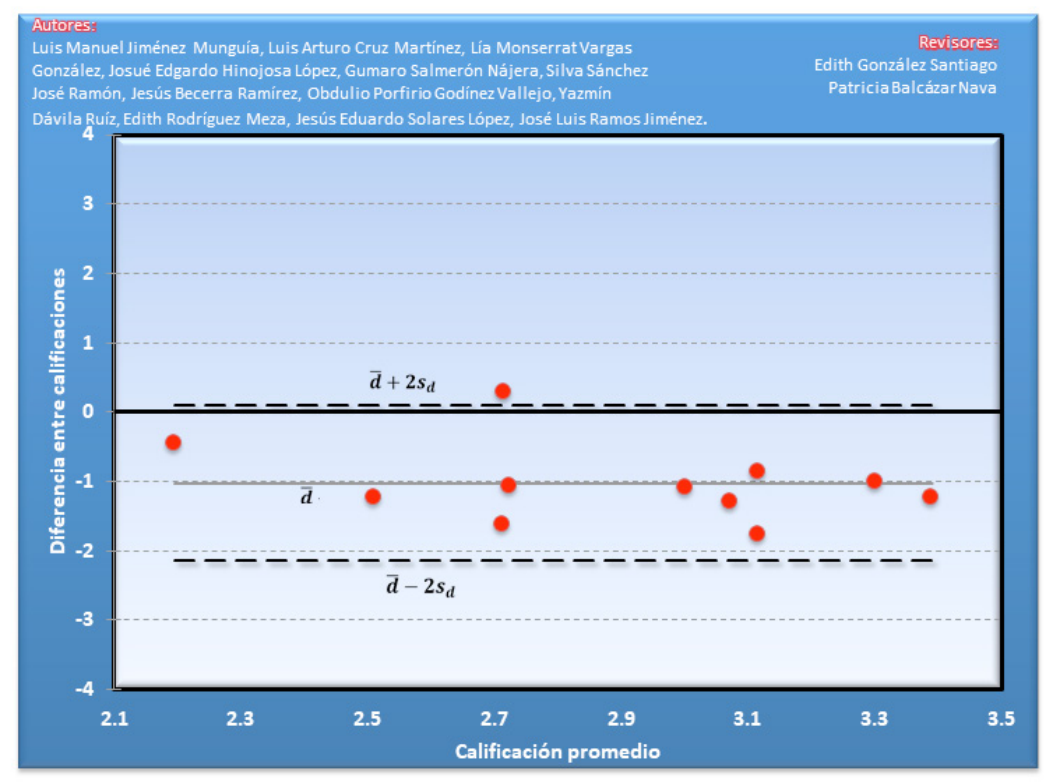

Índice de Acuerdo

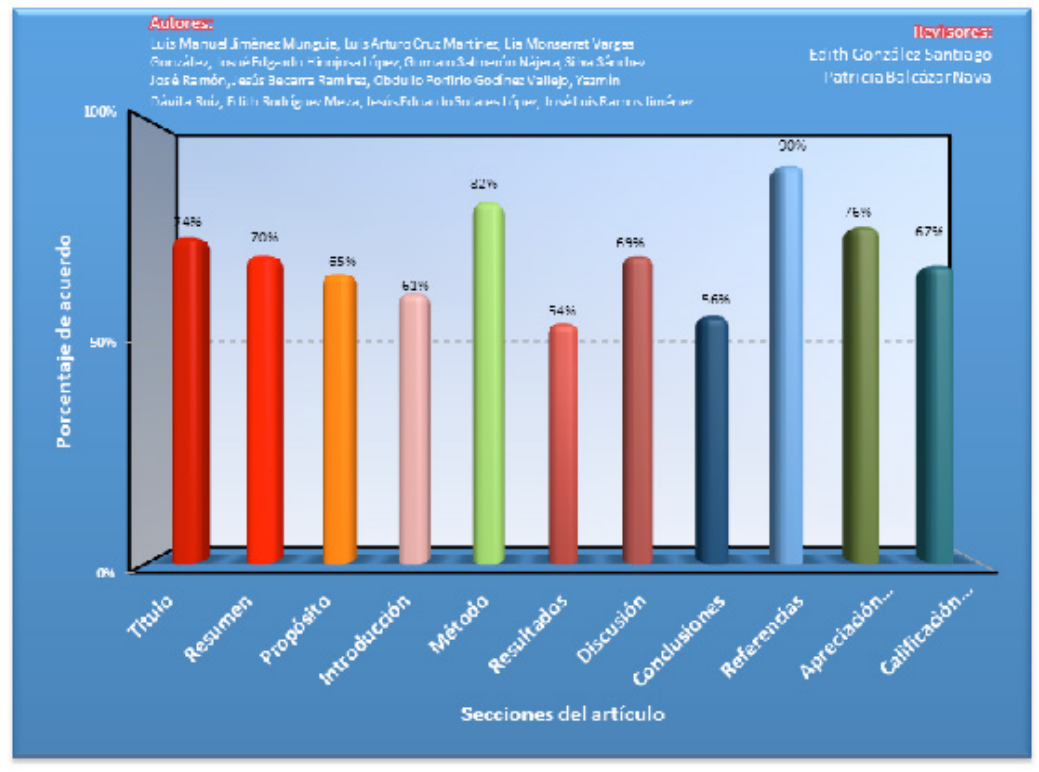




\begin{tabular}{|l|}
\hline Revisor 1 \\
\hline Edith González Santiago \\
\hline \\
\hline \\
El título no refleja las variables descritas en el objetivo \\
de la investigación ya que sólo menciona factor G de \\
inteligencia en mujeres sentenciadas, pero en el obje- \\
tivo menciona también conductas prosociales y con- \\
ductas de agresión. La población descrita en el objetivo \\
es "mujeres sentenciadas con trastornos psiquiátricos". \\
Sugiero una mayor claridad en el título referente a la \\
descripción de las variables incluidas en el estudio.
\end{tabular}

Debido a la temática y al tipo de manuscrito, que está planteando un estudio de tipo aplicado, en el cual se hizo evaluación del grupo antes y después, junto con una intervención y un formato de observación cualitativa, resulta de interés debido a que este tipo de estudios son difíciles de realizar y resultan un aporte que puede ayudar a mejorar las condiciones del grupo en el cual se aplicó o sirve como modelo para el diseño de futuras intervenciones. No obstante, en el título no está indicado, sólo cuando se lee, que se realizó la evaluación de conductas prosociales, por lo cual sería deseable que en el título se agregue esto. Si bien la redacción y el abordaje de cada uno de los aspectos que integran el trabajo de investigación son adecuados, es necesario que se ponga atención especial en el formato de citación, ya que muchas de las citas indicadas en el cuerpo del trabajo no están en la lista de referencias, lo cual es necesario cuidar; también es necesario cuidar el año de las citas, que no coincide con los de las referencias. Poner especial atención en el uso de números escritos completos o de ciertos términos y cifras que es necesario que estén redactados acorde con las normas del Manual de la APA. También es necesario seguir los términos de la APA para la escritura de citas cuando son varios autores (uso de et al.). Finalmente, es necesario revisar cuidadosamente la redacción de todo el trabajo y la lista de referencias, que deberá estar presentada conforme a las especificaciones de la APA en su Manual de Estilo de Publicación.

\section{Resumen}

Sugiero que se apegue a los criterios solicitados en cuanto a número de palabras y la redacción en párrafos (objetivo/hipótesis, diseño/metodología, resultados, conclusiones).
El resumen es claro y expone lo que se realizó, sólo falta incluir lo que es el diseño y excede el número de palabras permitidas tanto en inglés como en español 


\section{Revisor 1}

Revisor 2

Próposito del Estudio

Es necesaria la redacción de la pregunta de investigación o posible hipótesis, servirá de eje estructural para la discusión y conclusiones.
Se sugiere tanto en el título como en el apartado del método se destine un apartado donde quede con claridad expuesta la función de la variable de conductas prosociales, ya que pareciera desarticulada, cuando es parte importante de la investigación.

\section{Introducción}

Dado que existen estudios empíricos relacionados al tema de investigación, se sugiere la redacción de hipótesis para este estudio. También es necesario describir claramente las variables implicadas, por ejemplo, el tipo de trastornos psiquiátricos que se mencionan en la muestra, no están definidos. Lo mismo sucede con las conductas agresivas y las conductas prosociales que los autores mencionan en este estudio, pueden definirse operacionalmente.

Es necesario que se puntualice en el título y en el apartado del método la variable sobre conductas prosociales, que se defina con claridad esta variable y su función y forma de medirse y de incluirse en el diseño de investigación.

\section{Método}

Un criterio importante en la investigación científica es, sin duda, la replicación. Se sugiere que se describan puntualmente las sesiones realizadas en el estudio (cartas descriptivas), descripción detallada de la muestra (rango de edad, tipo de trastorno psiquiátrico) y el método para obtenerla. No se incluye el formato ni descripción de los registros observacionales ni de la entrevista semiestructurada. Se sugiere también, dado que se trata de una población vulnerable, la revisión de algún consejo o comité de ética de la institución.

Se comenta nuevamente la desarticulación de la variable de conductas prosociales con la otra variable, es necesario se especifique con cuidado cómo es que se realizó el registro de observación, quién lo realizó, cómo se procesó y y articular todo esto en un diseño donde quede insertada ésta, ya que al no estar incluso en el título, queda lugar a duda su evaluación en todo el estudio y por qué se le reporta.

\section{Resultados}

En el apartado de Resultados está descrita la muestra de forma detallada pero no así en el apartado de Participantes. Se sugiere la inclusión de las tablas en el texto de acuerdo a la APA.
Una vez que se incluya la variable de conductas prosociales en el cuerpo del trabajo, entonces queda claro en los resultados, la función de esta variable 


\begin{tabular}{|c|c|}
\hline $\begin{array}{l}\text { Revisor } 1 \\
\end{array}$ & Revisor 2 \\
\hline \multicolumn{2}{|c|}{ Discusión } \\
\hline - & $\begin{array}{l}\text { Cuidar detalles de redacción y del uso de citas que no es- } \\
\text { tán enlistadas en las referencias }\end{array}$ \\
\hline \multicolumn{2}{|c|}{ Conclusiones } \\
\hline $\begin{array}{l}\text { Se sugiere un mayor análisis de los resultados y las con- } \\
\text { clusiones para ampliar la discusión, principalmente so- } \\
\text { bre las limitaciones y alcances del estudio, así como las } \\
\text { aportaciones a las investigaciones realizadas en el tema } \\
\text { y las posibles aplicaciones en otros contextos. }\end{array}$ & Sección completa \\
\hline \multicolumn{2}{|c|}{ Referencias } \\
\hline $\begin{array}{l}\text { Es conveniente ampliar las referencias buscando fuen- } \\
\text { tes que fortalezcan el enfoque usado por los autores al } \\
\text { abordar la problemática expuesta, por ejemplo, no se } \\
\text { incluyen datos sobre las necesidades intelectuales o so- } \\
\text { ciales de poblaciones mexicanas en reclusión. }\end{array}$ & $\begin{array}{l}\text { Las referencias se presentan conforme a APA, pero no } \\
\text { coincide esta lista con las citas existentes. Muchas citas no } \\
\text { están incluidas en las referencias o no tienen el mismo año } \\
\text { y hay referencias que no se utilizaron en el cuerpo del tra- } \\
\text { bajo. Cuidar que se actualice la información al respecto, ya } \\
\text { que hay referencias antiguas que exceden los cinco años, } \\
\text { si bien algunas pueden ser clásicas, pero falta incluir más } \\
\text { recientes }\end{array}$ \\
\hline
\end{tabular}

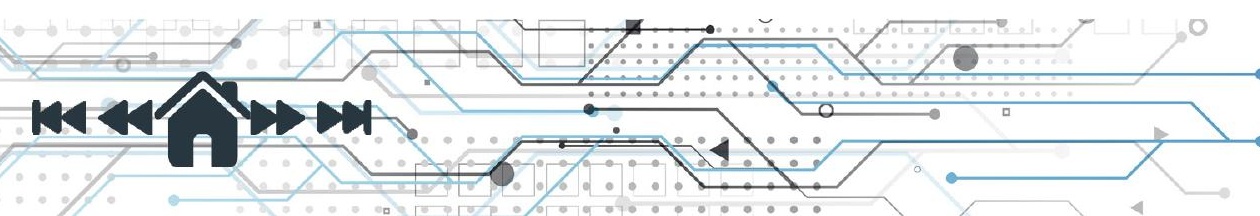


